\title{
Robotic vs. traditional stapler use in robotic portal anatomic lung resection
}

\author{
Joseph D. Phillips, Kayla A. Fay, Rian M. Hasson, Timothy M. Millington, David J. Finley \\ Section of Thoracic Surgery, Department of Surgery, Dartmouth-Hitchcock Medical Center, Lebanon, NH 03756, USA. \\ Correspondence to: Dr. Joseph D. Phillips, Thoracic Surgery, One Medical Center Dr, Lebanon, NH 03756, USA. \\ E-mail: joseph.d.phillips@hitchcock.org
}

How to cite this article: Phillips JD, Fay KA, Hasson RM, Millington TM, Finley DJ. Robotic vs, traditional stapler use in robotic portal anatomic lung resection. Mini-invasive Surg 2020;4:12. http://dx.doi.org/10.20517/2574-1225.2020.02

Received: 2 Jan 2020 First Decision: 19 Jan 2020 Revised: 27 Jan 2020 Accepted: 3 Feb 2020 Published: 14 Feb 2020

Science Editor: Noriyoshi Sawabata Copy Editor: Jing-Wen Zhang Production Editor: Tian Zhang

\begin{abstract}
Aim: Currently, there is a paucity of data comparing robotic to traditional video-assisted thoracic surgery stapling devices and the effects on perioperative outcomes during robotic anatomic lung resection. We sought to investigate our institutional experience with patients undergoing robotic anatomic lung resection stratified by the type of stapler used over a contemporary period.
\end{abstract}

Methods: We performed a retrospective review of a prospectively maintained thoracic surgery database and evaluated all patients who underwent robotic anatomic lung resection between January 2015 and December 2018. Patients were grouped based on the type of stapler used during surgery and preoperative characteristics and intraoperative and postoperative outcomes were compared.

Results: In total, 634 lung resections occurred during the study period. Of those, 236 met inclusion criteria, and 49 cases (20.8\%) fully utilized the robotic stapler. We found no clinically significant difference in preoperative or intraoperative characteristics between groups, except operative time was longer in the robot stapler group. This was likely related to surgeon learning curve. There were no differences between groups in postoperative outcomes or complications.

Conclusion: We found equivalent rates of complications, prolonged air leak, and chest tube duration between the two groups. Based on our data, we recommend that surgeons use the stapling device with which they are most confident.

Keywords: Robotic stapler, robotic lung resection, lung cancer, EndoWrist ${ }^{\circledR}$

\footnotetext{
(@) $\odot$

(C) The Author(s) 2020. Open Access This article is licensed under a Creative Commons Attribution 4.0 International License (https://creativecommons.org/licenses/by/4.0/), which permits unrestricted use, sharing, adaptation, distribution and reproduction in any medium or format, for any purpose, even commercially, as long as you give appropriate credit to the original author(s) and the source, provide a link to the Creative Commons license, and indicate if changes were made.
}

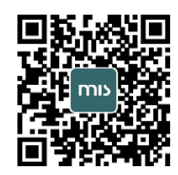




\section{INTRODUCTION}

Robotic resection for lung cancer is becoming increasingly accepted by the thoracic surgery community. Several recent publications have demonstrated the feasibility, safety, and equivalent oncologic outcomes for robotic anatomic resections compared to traditional Video-Assisted Thoracic Surgery (VATS) and improved postoperative outcomes compared to traditional thoracotomy ${ }^{[1-6]}$. Advantages of robotic resection over traditional VATS include improved visualization with three-dimensional viewing, articulated instruments, and increased flexibility in areas of limited operating space. Previous drawbacks to robotics have required an experienced bedside assistant for division of the hilar structures with a traditional VATS stapler, or for the operating surgeon to leave the console to return to the bedside to perform this critical portion of the operation. In 2014, the da Vinci Xi System (Intuitive Surgical, Sunnyvale CA) was introduced, with instrument updates in early 2016 which provided a $30-\mathrm{mm}$ curved-tip stapler that was capable of providing the console surgeon the ability to control and fire staplers for division of vascular, bronchial, and parenchymal structures ${ }^{[7,8]}$. This decreased some of the potential limitations for surgeons to perform minimally invasive anatomic lung resections by allowing a critical step to be placed back in the hands of the operating surgeon at the console ${ }^{[0]}$.

Currently, there is a paucity of data regarding the perioperative outcomes of robotic anatomic lung resection comparing robotic staplers to traditional VATS stapling devices. We sought to investigate our institutional experience with patients undergoing robotic anatomic lung resection stratified by the type of stapler used over a contemporary period.

\section{METHODS}

\section{Patients}

A retrospective analysis of an institutional review board approved prospective Thoracic Surgery database was performed. All consecutive patients who underwent lung resection between 1 January 2015 and 31 December 2018 were included. Patients were excluded if they underwent a non-anatomic resection (wedge), underwent planned or were converted to a thoracotomy, or had a VATS that did not include the use of the da Vinci robotic system [Figure 1]. The primary aim of this study was to investigate intraoperative and postoperative outcomes with the da Vinci EndoWrist ${ }^{\circ}$ robotic stapler compared to the Covidien Endo GIA $^{\text {TM }}$ stapler (Medtronic, Fridley MN) in robotic anatomic lung resections. This study was approved by the Committee for the Protection of Human Subjects (\#30040).

\section{Data collection}

Demographic data (age, sex, and race), pulmonary co-morbidities, operative data (operative time and stapler use), pathologic data (stage and lymph nodes collected), postoperative length of stay (LOS), and 30-day complications were obtained. Operative time, in minutes, was calculated from surgery start and stop times. Postoperative complications were monitored for 30 days from the index procedure date and graded I-IV as classified by Clavien-Dindo ${ }^{[10,11]}$. The primary outcome of interest was presence of a postoperative prolonged air leak (PAL), which was defined as an air leak lasting more than five days, as defined by the Society of Thoracic Surgeons ${ }^{[12]}$.

\section{Surgical technique}

Anatomic lung resections were performed by two surgeons as previously described ${ }^{[13]}$. Briefly, all resections utilized the da Vinci Xi system with a four-arm technique and an additional 15-mm assistant port. The camera and robotic ports are placed in the 6th-8th intercostal spaces, depending on the tumor site. The assistant port is placed as low as possible without traversing the diaphragm. When a traditional VATS stapler is used, $8-\mathrm{mm}$ robotic trocars are placed and the stapler is introduced via the $15-\mathrm{mm}$ assistant port. When the robotic stapler is used, one or two $12-\mathrm{mm}$ trocars are placed, as described previously ${ }^{[7]}$. There are limited requirements for the assistant to change instruments when the robotic stapler is used, but he/she 


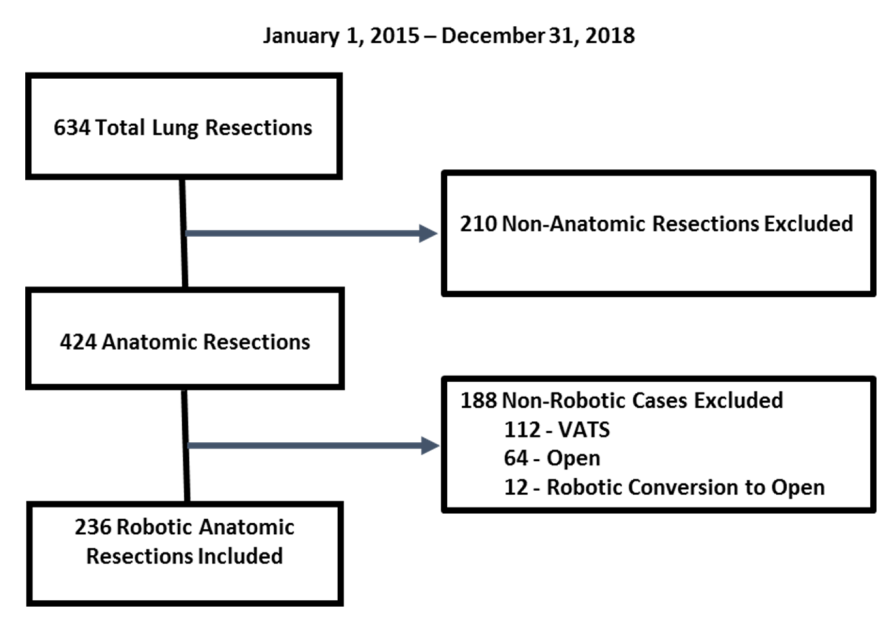

Figure 1. Study inclusion and exclusion. VATS: video-assisted thoracic surgery

maintains the ability to insert ancillary instruments and remove specimens without undocking a robotic arm. In addition, retraction and tension are controlled by the surgeon and exposure of the operative field is more stable $\mathrm{e}^{[1,15]}$. Typically, a bipolar grasper is used in the surgeon's left hand and a monopolar spatula in the right. Retraction is facilitated via a tip-up fenestrated grasper in the $3 \mathrm{rd}$ arm. The spatula provides excellent blunt dissection capability, has less arc than the hook, and is less sharp than the Maryland bipolar dissector. A mediastinal lymph node dissection is performed initially, as it provides exposure for portions of the bronchial and lobar lymph node dissections. The pulmonary artery in the fissure is then dissected as appropriate, limiting the dissection of lung parenchyma as much as possible. The hilar structures and lymph nodes are then circumferentially dissected. The vascular structures are often divided first, followed by the bronchus. Any remaining lung parenchyma is divided at convenient points to facilitate exposure.

\section{Stapler}

Stapler choice was at the discretion of the attending surgeon. Intuitive released the $30-\mathrm{mm}$ curved EndoWrist ${ }^{\oplus}$ robotic stapler in early 2016 and the first use of this stapler at our institution occurred in September 2016. Robotic stapler use was exclusively performed by one surgeon (JDP). Typically, division of structures by staple load were: vascular (white), bronchus (green), and parenchyma (blue or green based on thickness). Hilar structures are typically divided with the $30-\mathrm{mm}$ curved stapler and parenchyma with the $45-\mathrm{mm}$ stapler. The Covidien Endo GIA ${ }^{\mathrm{TM}} 12-\mathrm{mm}$ stapler with Tri-Staple ${ }^{\mathrm{TM}} 2.0$ Intelligent Reload technology was used during the study period. Typically, division of structures by staple load were: vascular ( $\tan )$, bronchus (purple), and parenchyma (tan, purple, or black based on thickness).

\section{Analysis}

Univariate analysis was performed to assess for differences in perioperative, intraoperative, and postoperative characteristics between the cases that utilized the EndroWrist ${ }^{\bullet}$ robotic stapler and those that utilized the Endo GIA ${ }^{\mathrm{TM}}$ stapler. Two-tailed student's $t$-tests were used for continuous variables and chisquare tests were used for categorical variables. A $P$-value of $<0.05$ was considered statistically significant.

\section{RESULTS}

In total, 634 lung resections occurred during the study period. Of those, 236 met inclusion criteria, and 49 cases $(20.8 \%)$ utilized the robotic stapler fully. Three cases used the robotic stapler for division of the hilar structures but the Covidien stapler for division of the lung parenchyma. These three cases were classified in the Covidien stapler group. Of note, only 12 planned robotic cases were converted to open and were excluded, corresponding to a conversion rate of $4.8 \%$. Of these 12 conversions: three were following 
Table 1. Characteristics of study population

\begin{tabular}{llll}
\hline & $\begin{array}{c}\text { Robotic stapler } \\
\boldsymbol{n}=\mathbf{4 9}\end{array}$ & $\begin{array}{c}\text { Covidien stapler } \\
\boldsymbol{n}=\mathbf{1 8 7}\end{array}$ & $\boldsymbol{P}^{\text {-value }}{ }^{\mathbf{1}}$ \\
\hline Age, Mean (SD) & $67.2(8.3)$ & $67.0(9.0)$ & 0.89 \\
Male, (\%) & $25(51.0)$ & $77(41.2)$ & 0.22 \\
Caucasian, (\%) & $49(100)$ & $184(98.4)$ & $\times$ \\
BMI, Mean (SD) & $28.4(6.6)$ & $27.0(5.8)$ & 0.14 \\
Pack years, Mean (SD) & $46.9(24.6)$ & $43.2(29.2)$ & 0.47 \\
Smoking status ${ }^{2}$, (\%) & & & \\
Never & $9(18.4)$ & $24(12.8)$ & 0.32 \\
Former & $29(59.2)$ & $120(64.2)$ & 0.52 \\
Current & $11(22.4)$ & $43(23.0)$ & 0.94 \\
Pulmonary co-morbidities, (\%) & & & \\
Asthma & $7(14.3)$ & $11(5.9)$ & 0.05 \\
COPD & $14(28.6)$ & $49(26.2)$ & 0.74 \\
Pulmonary hypertension & $1(2.0)$ & $2(1.1)$ & 0.59 \\
Emphysema & $1(2.0)$ & $4(2.1)$ & 0.97 \\
None & $28(57.1)$ & $120(64.2)$ & 0.37 \\
Pulmonary function, Mean (SD) & & & \\
FEV1 (L) & $2.24(0.6)$ & $2.12(0.7)$ & 0.23 \\
FEV1\% predicted & $84.2(16.0)$ & $80.1(18.3)$ & 0.15 \\
FVC (L) & $3.3(0.9)$ & $3.16(0.9)$ & 0.25 \\
FVC\% predicted & $91.2(17.9)$ & $91.2(17.2)$ & 1.0 \\
Induction therapy, (\%) & $5(10.2)$ & $18(9.6)$ & 0.90 \\
\hline
\end{tabular}

${ }^{1} P$-values from student's $t$-test or chi-square test where appropriate. ${ }^{2}$ Classified at time of first consultation with a thoracic surgeon. SD: standard deviation; BMI: body mass index; COPD: chronic obstructive pulmonary disease; FEV1: forced expiratory volume in 1 second; FVC: forced vital capacity; $x$ : statistics unable to be performed

induction therapy, two required a pulmonary artery plasty, and seven were related to a combination of adhesions or tumor location that limited safe dissection around critical structures. Table 1 provides a comparison of the perioperative patient characteristics between the robotic and traditional stapler groups. There was no difference in demographics between the two groups, with a mean age of 67 in both and most patients were Caucasian. The robotic stapler group had more patients with a history of asthma, $(14.2 \% v s$. $5.9 \%, P=0.05)$, but otherwise did not differ in the presence of other co-morbidities. In addition, there were no differences in preoperative pulmonary function testing or rate of induction therapy.

Intraoperative characteristics between the two groups are compared in Table 2. Cases that utilized the robotic stapler had a significantly longer average operative time (224 $\min v s .176 \mathrm{~min}, P<0.001)$. Given that these cases were performed by a surgeon in the first few years of practice, this likely reflects a learning curve rather than inherent delay with use of the robotic stapler, as evidenced by a significant decrease in average operative time from 2016-2017 $(n=21)$ to $2018(n=28)$ (247 min vs. $207 \mathrm{~min}$, respectively; $P=0.01$ ). There was no difference in the average number of staple loads used per case between the two groups. While the number of staple loads may seem high, anatomic resection is often preceded by a diagnostic wedge, which obviously increases the total number of staple loads used. Pathologic staging was similar between the two groups, although there were significantly more stage IIB cases in the robotic stapler group. There were no differences in lymph node stations or total lymph nodes collected between groups.

Postoperative outcomes are compared in Table 3. There was no difference in average LOS between the two groups (median three days for both). Median chest tube duration was two days for both groups, and $20 \%$ of patients in each group were discharged with a chest tube. The overall postoperative PAL rate was $25.8 \%$ for the entire cohort. Within the robotic stapler group, the PAL rate was $20.4 \%$, compared to $27.3 \%$ in the Covidien stapler group $(P=0.33)$. In the robotic stapler group, one patient with a PAL underwent a bedside doxycycline pleurodesis. In the Covidien stapler group, 10 patients underwent a procedure for management (six had bedside doxycycline pleurodesis, three had endobronchial valves, and one had both bedside 
Table 2. Operative characteristics of study population

\begin{tabular}{llll}
\hline & $\begin{array}{c}\text { Robotic stapler } \\
\boldsymbol{n = 4 9}\end{array}$ & $\begin{array}{c}\text { Covidien stapler } \\
\boldsymbol{n}=\mathbf{1 8 7}\end{array}$ & $\boldsymbol{P}_{\text {-value }}{ }^{\mathbf{1}}$ \\
\hline Operative time, minutes, Mean (SD) & $224(55)$ & $176(48)$ & $<0.001$ \\
Number of staple loads', Mean (SD) & $11.0(3.8)$ & $10.1(3.6)$ & 0.11 \\
Resection type, (\%) & & & \\
Segment & $2(4.1)$ & $17(9.1)$ & 0.25 \\
Lobe & $46(93.9)$ & $168(89.8)$ & 0.39 \\
Bi-Lobe & $1(2.0)$ & $2(1.1)$ & 0.59 \\
Tumor location, (\%) & & & \\
Right upper lobectomy & $18(36.7)$ & $62(33.2)$ & 0.64 \\
Right middle lobectomy & $4(8.2)$ & $13(7.0)$ & 0.77 \\
Right lower lobectomy & $8(16.3)$ & $33(17.6)$ & 0.83 \\
Left upper lobectomy & $12(24.5)$ & $48(25.7)$ & 0.87 \\
Left lower lobectomy & $6(12.2)$ & $29(15.5)$ & 0.57 \\
Bi-lobectomy (Middle/Lower) & $1(2.0)$ & $2(1.1)$ & 0.59 \\
Pathologic stage & & & \\
IA & $25(51.0)$ & $83(44.4)$ & 0.41 \\
IB & $10(20.4)$ & $42(22.5)$ & 0.76 \\
IIA & $1(2.0)$ & $9(4.8)$ & 0.39 \\
IIB & $8(16.3)$ & $10(5.3)$ & 0.01 \\
IIIA & $2(4.1)$ & $18(9.6)$ & 0.21 \\
IIIB & $1(2.0)$ & $1(0.5)$ & 0.31 \\
IV & 0 & $6(3.2)$ & $\times$ \\
Other & $2(4.1)$ & $18(9.6)$ & 0.21 \\
Lymph nodes, Mean (SD) & $14.1(6.1)$ & $14.7(7.2)$ & 0.58 \\
Total collected & $7.7(4.0)$ & $7.7(5.2)$ & 0.98 \\
N1 collected & $6.3(3.3)$ & $7.0(4.2)$ & 0.31 \\
N2 collected & $49(100)$ & $184(98.4)$ & $\times$ \\
Margin status, (\%) & $3(1.6)$ & $\times$ \\
RO & 0 & & \\
R1 & & & \\
\hline & & & \\
\hline
\end{tabular}

${ }^{1} P$-values from student's t-test or chi-square test where appropriate. ${ }^{2}$ Excluded eight cases for insufficient staple load number data (seven Covidien and one robotic). SD: standard deviation; $x$ : statistics unable to be performed

Table 3. Postoperative characteristics of study population

\begin{tabular}{|c|c|c|c|}
\hline & $\begin{array}{c}\text { Robot stapler } \\
n=49\end{array}$ & $\begin{array}{l}\text { Covidien stapler } \\
\quad n=187\end{array}$ & $\boldsymbol{P}_{\text {-value }}$ \\
\hline Length of stay, days, Median (range) & $3(1-14)$ & $3(1-40)$ & 0.16 \\
\hline Discharged with a chest tube, (\%) & $10(20.4)$ & $40(21.4)$ & 0.88 \\
\hline $\begin{array}{l}\text { Chest tube duration, days, Median } \\
\text { (range) }\end{array}$ & $2(1-23)$ & $2(1-43)$ & 0.17 \\
\hline Grade $3 / 4$ complication rate ${ }^{2}$, (\%) & $6(12.2)$ & $29(15.5)$ & 0.57 \\
\hline \multicolumn{4}{|l|}{ Complications $^{3},(\%)$} \\
\hline Return to operative room ${ }^{4}$ & $1(2.0)$ & $1(0.5)$ & 0.31 \\
\hline Transfusion & $1(2.0)$ & $3(1.6)$ & 0.83 \\
\hline Prolonged air leak ${ }^{5}$ & $10(20.4)$ & $51(27.3)$ & 0.33 \\
\hline Pneumonia & $2(4.1)$ & $16(8.6)$ & 0.29 \\
\hline Pleural effusion ${ }^{6}$ & $1(2.0)$ & $5(2.7)$ & 0.80 \\
\hline Atelectasis $^{7}$ & $1(2.0)$ & $1(0.5)$ & 0.31 \\
\hline Pneumothorax ${ }^{8}$ & 0 & $10(5.3)$ & x \\
\hline Atrial fibrillation ${ }^{9}$ & 0 & $13(7.0)$ & $x$ \\
\hline Myocardial infarction & 0 & 0 & $x$ \\
\hline Readmission ${ }^{3},(\%)$ & $5(10.2)$ & $25(13.4)$ & 0.32 \\
\hline Pneumonia & $2(4.1)$ & $7(3.7)$ & 0.91 \\
\hline Pneumothorax & 0 & $5(2.7)$ & $x$ \\
\hline Pleural effusion & $1(2.0)$ & $1(0.5)$ & 0.31 \\
\hline Empyema & 0 & $1(0.5)$ & $x$ \\
\hline Infected pleural space & 0 & $1(0.5)$ & $x$ \\
\hline
\end{tabular}




\begin{tabular}{llll} 
Atrial fibrillation & 0 & $2(1.1)$ & $x$ \\
Other $^{10}$ & $2(4.1)$ & $7(3.7)$ & 0.91 \\
30 -day mortality, (\%) & 0 & $1(0.5)$ & $x$ \\
\hline
\end{tabular}

${ }^{1} P$-values from student's t-test or chi-square test where appropriate. ${ }^{2}$ Grade $3 / 4$ complication as classified by Clavien-Dindo. ${ }^{3}$ Within 30-days of index procedure. ${ }^{4}$ Unexpected return to OR within 30 -days of index procedure. ${ }^{5}$ Defined as an air leak that lasted beyond postoperative day $5 .{ }^{6}$ Requiring drainage. ${ }^{7}$ Requiring bronchoscopy. ${ }^{8}$ Requiring chest tube reinsertion. ${ }^{9}$ Requiring treatment. ${ }^{10}$ Includes anemia, bowel obstruction, dehydration, syncope, hyponatremia, gastrointestinal bleed, fluid overload, and thrombus. x: statistics unable to be performed

pleurodesis and endobronchial valves). There was no difference in grade $\geq 3$ complications, readmissions, or 30-day mortality.

\section{DISCUSSION}

As new technology becomes available, it is important that surgeons critically evaluate its use. The 30-mm curve tip EndoWrist ${ }^{\circledast}$ stapler was introduced in March 2016. However, only a few reports to date in the literature describe its use in pulmonary resections ${ }^{[7,8,16,17]}$. To our knowledge, the current study is the first to directly compare the robotic stapler and a traditional VATS stapler related to perioperative outcomes in robotic anatomic lung resections. We found no clinically significant differences in preoperative characteristics between the two stapler groups at our institution. There were also no clinically significant differences noted in the number of staple loads used, pathologic stage, or lymph nodes harvested. We did identify a significant increase in operative time in the group that utilized the robotic stapler. As the robotic stapler was exclusively used by a new attending surgeon, this likely represents a learning curve rather than an intrinsic delay related to stapler use, as evidenced by the significant reduction in operative time for these cases over the course of the study period. Moreover, there were no differences in LOS, chest tube duration, or postoperative complications between the two groups. Overall, our outcomes are in-line with recently published experiences ${ }^{[6,8,18,19]}$.

Ultimately, we did not find a difference in the rate of postoperative PAL or chest tube duration between the two groups. While a recent analysis of the Society of Thoracic Surgeons DataBase reported an overall rate of PAL of $10.4 \%{ }^{[20]}$, rates following anatomic lung resection range from $6 \%$ to $30 \%{ }^{[19]}$. Several risk factors have been reported to increase the risk of PAL, including forced expiratory volume in 1 second $<70 \%$ of predicted, body mass index $<25 \mathrm{~kg} / \mathrm{m}^{2}$, previous smoking, anatomic lung resection, pleural adhesions, male sex, and right upper lobe procedure ${ }^{[19,20]}$. Many of our patients have several, if not most of these risk factors. In addition, our rural patient population has a significant proportion of patients who began smoking at an early age. Smoking in childhood and during the teenage years can slow lung development and increase the risk of chronic obstructive pulmonary disease in adulthood ${ }^{[21]}$. Early smoke exposure leads to airway inflammation and parenchymal lung injury with larger saccules, increased density of interstitial tissue, and reduced elastin and collagen ${ }^{[22]}$. These factors may help to explain our rate of postoperative PAL in the setting of otherwise low rates of complications. However, our study is not powered or intended to predict an increase in PAL based on these factors. In addition, we are aggressive about discharging patients from the hospital with a chest tube in place. Given our rural catchment area, this may result in some delay in actual chest tube removal beyond Postoperative Day 5 when an air leak is not actually present.

Variability in the techniques of robotic anatomic lung resection exist. A recently published survey of highvolume robotic thoracic surgeons demonstrated that most respondents utilized a four-arm approach and $94 \%$ used an additional non-robotic assistant port ${ }^{[23]}$. In respondents, there was not a universal standard port placement, and stapling port strategies were nuanced by lobe and type of stapler used. As additional technologies are developed, it will be important to evaluate their efficacy and effectiveness, in terms of both clinical outcomes and healthcare costs. 
The successful performance of robotic lung resection requires a strong team in the operating room composed of surgeons, nurses, surgical techs, anesthesia providers, and a bedside assistant. The literature describes the learning curve of a robotic lobectomy as 18-32 cases for a surgeon and 20 for a bedside assistant ${ }^{[24-26]}$. Specific to anatomic lung resection, division of the pulmonary vascular structures is a potentially hazardous portion of the operation that requires significant skill to perform safely. Prior to the development of the robotic stapler, this required a competent bedside assistant or the console surgeon to return to the bedside. At our institution, we have dedicated physician assistants or trained residents who can safely complete these tasks. However, this may not be the case for every thoracic surgeon. Others have fully described the range of motion capabilities of the EndoWrist ${ }^{\oplus}$ stapler, as well as the safety components that ensure adequate closing and prevent the firing of an incorrectly loaded or spent reload ${ }^{[8]}$. Drawbacks of using the robotic stapler are the need for a $12-\mathrm{mm}$ port, the long length of the stapler load that can impede maneuverability in the chest, and the rotational limitation that can occur when the wrist is fully flexed. This stapler does provide the console surgeon with the ability to control the stapler during division of critical structures and may improve one's ability to perform complex minimally invasive techniques with reduced conversions ${ }^{[9,17]}$. These benefits may be more apparent at sites where a fully thoracic-trained bedside assistant is not available.

The findings of our study should be viewed in the context of several limitations. This is a retrospective, single institution cohort study and subject to potential selection bias, and our results may not be generalizable to other patient populations. In addition, our data show that the robotic stapler group operating time was significantly longer. However, as mentioned above, this is likely related to one surgeon's learning curve and not an inherently longer time for use of the stapler. Nevertheless, our outcomes are inline or better than those reported by multiple authors in the literature, and, to our knowledge, this is the first study to directly compare the EndoWrist ${ }^{\circledR}$ robotic stapler to a traditional Endo GIA ${ }^{\mathrm{TM}}$ stapler. Clinical outcomes appear to be equivalent in our patient population and further study is needed to assess if there is a difference in cost-effectiveness between these devices.

In conclusion, robotic anatomic lung resection has been shown to be safe and feasible with equivalent long-term oncologic outcomes when compared to VATS and thoracotomy. In this study, we compared perioperative outcomes of patients undergoing robotic anatomic lung resection to assess whether there are any differences based on the type of stapler utilized. We found equivalent rates of complications, PAL, and chest tube duration between the two groups. Based on our data, we recommend that surgeons use the stapling device with which they are most confident.

\section{DECLARATIONS}

\section{Authors' contributions}

Made substantial contributions to conception and design of the study and performed data analysis and interpretation: Phillips JD, Fay KA, Finley DJ

Made substantial contributions to data interpretation and drafting and critical revisions of the manuscript: Phillips JD, Fay KA, Hasson RM, Millington TM, Finley DJ

\section{Availability of data and materials}

The data source is a prospectively collected institutional database containing personal health information (PHI). Per Dartmouth-Hitchcock Medical Center (DHMC) policy, any request for data would require an approved Data Use Agreement (DUA) between DHMC and the requesting individual and/or institution.

\section{Financial support and sponsorship}

None. 


\section{Conflicts of interest}

Phillips JD has previously received consulting fees from Intuitive Surgical, Inc. but has no ongoing relationship. All other authors declared that there are no conflicts of interest related to this work.

\section{Ethical approval and consent to participate}

The study was reviewed by the Committee for the Protection of Human Subjects of Dartmouth-Hitchcock Medical Center and approved.

\section{Consent for publication}

Not applicable.

\section{Copyright}

(c) The Author(s) 2020.

\section{REFERENCES}

1. Zirafa C, Aprile V, Ricciardi S, Romano G, Davini F, et al. Nodal upstaging evaluation in NSCLC patients treated by robotic lobectomy. Surg Endosc 2019;33:153-8.

2. Toker A, Özyurtkan MO, Demirhan O, Ayalp K, Kaba E, et al. Lymph node dissection in surgery for lung cancer: comparison of open vs. video-assisted vs. robotic-assisted approaches. Ann Thorac Cardiovasc Surg 2016;22:284-90.

3. Liang H, Liang W, Zhao L, Chen D, Zhang J, et al. Robotic versus video-assisted lobectomy/segmentectomy for lung cancer: a metaanalysis. Ann Surg 2018;268:254-9.

4. Cerfolio RJ, Ghanim AF, Dylewski M, Veronesi G, Spaggiari L, et al. The long-term survival of robotic lobectomy for non-small cell lung cancer: a multi-institutional study. J Thorac Cardiovasc Surg 2018;155:778-86.

5. Zirafa CC, Cavaliere I, Ricciardi S, Romano G, Davini F, et al. Long-term oncologic results for robotic major lung resection in nonsmall cell lung cancer (NSCLC) patients. Surg Oncol 2019;28:223-7.

6. Kneuertz PJ, D'Souza DM, Richardson M, Abdel-Rasoul M, Moffatt-Bruce SD, et al. Long-term oncologic outcomes after robotic lobectomy for early-stage non-small-cell lung cancer versus video-assisted thoracoscopic and open thoracotomy approach. Clin Lung Cancer 2019; Epub ahead of print [PMID: 31685354 DOI: 10.1016/j.cllc.2019.10.004]

7. Pearlstein DP. Robotic lobectomy utilizing the robotic stapler. Ann Thorac Surg 2016;102:e591-3.

8. Galetta D, Casiraghi M, Pardolesi A, Borri A, Spaggiari L. New stapling devices in robotic surgery. J Vis Surg 2017;3:45.

9. Kim MP, Nguyen DT, Meisenbach LM, Graviss EA, Chan EY. Da Vinci Xi robot decreases the number of thoracotomy cases in pulmonary resection. J Thorac Dis 2019;11:145-53.

10. Dindo D, Demartines N, Clavien PA. Classification of surgical complications: a new proposal with evaluation in a cohort of 6336 patients and results of a survey. Ann Surg 2004;240:205-13.

11. Clavien PA, Barkun J, de Oliveira ML, Vauthey JN, Dindo D, et al. The Clavien-Dindo classification of surgical complications: fiveyear experience. Ann Surg 2009;250:187-96.

12. STS National Database 2018. General thoracic surgery database training manual. Available from: https://www.sts.org/sites/default/files/ content/GTSDTrainingManual_September24_2018.pdf [Last accessed on 11 Feb 2020]

13. Phillips JD, Finley DJ. Editorial on expanded indications for robotic surgery in stage IIIA non-small cell lung cancer. J Thorac Dis 2019;11 (Suppl 9):S1172-4.

14. Veronesi G, Galetta D, Maisonneuve P, Melfi F, Schmid RA, et al. Four-arm robotic lobectomy for the treatment of early-stage lung cancer. J Thorac Cardiovasc Surg 2010;140:19-25.

15. Cerfolio RJ. Total port approach for robotic lobectomy. Thorac Surg Clin 2014;24:151-6.

16. Kim MP, Chan EY. "Five on a dice" port placement for robot-assisted thoracoscopic right upper lobectomy using robotic stapler. J Thorac Dis 2017;9:5355-62.

17. Khan N, Fikfak V, Chan EY, Kim MP. "Five on a dice" port placement allows for successful robot-assisted left pneumonectomy. Thorac Cardiovasc Surg Rep 2017;6:e42-4.

18. Guo F, Ma D, Li S. Compare the prognosis of Da Vinci robot-assisted thoracic surgery (RATS) with video-assisted thoracic surgery (VATS) for non-small cell lung cancer: a meta-analysis. Medicine (Baltimore) 2019;98:e17089.

19. Attaar A, Tam V, Nason KS. Risk factors for prolonged air leak after pulmonary resection: a systematic review and meta-analysis. Ann Surg 2019; Epub ahead of print [PMID: 31577547 DOI: 10.1097/SLA.0000000000003560]

20. Seder CW, Basu S, Ramsay T, Rocco G, Blackmon S, et al. A Prolonged air leak score for lung cancer resection: an analysis of the society of thoracic surgeons general thoracic surgery database. Ann Thorac Surg 2019;108:1478-83.

21. Centers for Disease Control and Prevention Office on Smoking and Health. A report of the surgeon general. preventing tobacco use among youth and young adults: we can make the next generation tobacco-free. Available from: https://www.cdc.gov/tobacco/data statistics/sgr/2012/consumer_booklet/index.htm [Last accessed on 11 Feb 2020]

22. Lampalo M, Majer M, Jukić I, Stanić HS, Barišić B, et al. Health sequelae of tobacco exposure in childhood and adolescence. Psychiatr Danub 2019;31 (Suppl 1):39-43. 
23. Oh DS, Tisol WB, Cesnik L, Crosby A, Cerfolio RJ. Port strategies for robot-assisted lobectomy by high-volume thoracic surgeons: a nationwide survey. Innovations (Phila) 2019;14:545-52.

24. Song G, Sun X, Miao S, Li S, Zhao Y, et al. Learning curve for robot-assisted lobectomy of lung cancer. J Thorac Dis 2019;11:2431-7.

25. Arnold BN, Thomas DC, Bhatnagar V, Blasberg JD, Wang Z, et al. Defining the learning curve in robot-assisted thoracoscopic lobectomy. Surgery 2019;165:450-4.

26. Meyer M, Gharagozloo F, Tempesta B, Margolis M, Strother E, et al. The learning curve of robotic lobectomy. Int J Med Robot 2012;8:448-52. 